\title{
Muscular Responses and Movement Strategies During Stumbling Over Obstacles
}

\author{
A. M. SCHILLINGS,,${ }^{1,2}$ B.M.H. VAN WEZEL, ${ }^{1}$ TH. MULDER,,${ }^{2,3}$ AND J. DUYSENS ${ }^{1}$ \\ ${ }^{1}$ Department of Medical Physics and Biophysics, University of Nijmegen, 6525 EZ Nijmegen; ${ }^{2}$ Sint Maartenskliniek \\ Research, 6500 GM Nijmegen; and ${ }^{3}$ Institute of Neurology, University Hospital Nijmegen, 6500 HB Nijmegen, \\ The Netherlands
}

\begin{abstract}
Schillings, A. M., B.M.H. Van Wezel, Th. Mulder, and J. Duysens. Muscular responses and movement strategies during stumbling over obstacles. J. Neurophysiol. 83: 2093-2102, 2000. Although many studies have investigated reflexes after stimulation of either cutaneous or proprioceptive afferents, much less is known about responses after more natural perturbations, such as stumbling over an obstacle. In particular, the phase dependency of these responses and their relation to the stumbling behavior has received little attention. Hence response strategies during stumbling reactions after perturbations at different times in the swing phase of gait were studied. While subjects walked on a treadmill, a rigid obstacle unexpectedly obstructed the forward sway of the foot. All subjects showed an "elevating strategy" after early swing perturbations and a "lowering strategy" after late swing perturbations. During the elevating strategy, the foot was directly lifted over the obstacle through extra knee flexion assisted by ipsilateral biceps femoris (iBF) responses and ankle dorsiflexion assisted by tibialis anterior (iTA) responses. Later, large rectus femoris (iRF) activations induced knee extension to place the foot on the treadmill. During the lowering strategy, the foot was quickly placed on the treadmill and was lifted over the obstacle in the subsequent swing. Foot placement was actively controlled by $\mathrm{iRF}$ and iBF responses related to knee extension and deceleration of the forward sway. Activations of iTA mostly preceded the main ipsilateral soleus (iSO) responses. For both strategies, four response peaks could be distinguished with latencies of $\sim 40 \mathrm{~ms}$ (RP1), $75 \mathrm{~ms}$ (RP2), $\sim 110 \mathrm{~ms}$ (RP3), and $\sim 160$ $\mathrm{ms}$ (RP4). The amplitudes of these response peaks depended on the phase in the step cycle. The phase-dependent modulation of the responses could not be accounted for by differences in stimulation or in background activity and therefore is assumed to be premotoneuronal in origin. In mid swing, both the elevating and lowering strategy could occur. For this phase, the responses of the two strategies could be compared in the absence of phase-dependent response modulation. Both strategies had the same initial electromyographic responses till $\sim 100 \mathrm{~ms}(\mathrm{RP} 1-\mathrm{RP} 2)$ after perturbation. The earliest response (RP1) is assumed to be a short-latency stretch reflex evoked by the considerable impact of the collision, whereas the second (RP2) has features reminiscent of cutaneous and proprioceptive responses. Both these responses did not determine the behavioral response strategy. The functionally important response strategies depended on later responses (RP3-RP4). These data suggest that during stumbling reactions, as a first line of defense, the CNS releases a relatively aspecific response, which is followed by an appropriate behavioral response to avoid the obstacle.
\end{abstract}

\section{N T R O D U C T I O N}

The pattern and timing of motor output during human locomotion are determined by a mixture of influences, some arising

The costs of publication of this article were defrayed in part by the payment of page charges. The article must therefore be hereby marked "advertisement" in accordance with 18 U.S.C. Section 1734 solely to indicate this fact. from neural circuits entirely within the CNS and others arising from a variety of sensory afferents. The electromyographic (EMG) responses in leg muscles occurring after stimulation of cutaneous and proprioceptive afferents during locomotion have been described in many studies (see reviews Dietz 1992; Duysens et al. 2000). The amplitudes of the responses to such stimuli were dependent on the phase (or time) of stimulation in the step cycle. For instance, electrical stimulation of the human sural nerve yields facilitation of the ankle flexor muscle tibialis anterior during early swing, but leads to suppression when delivered during late swing (reflex reversal) (Duysens et al. 1990, 1992, 1996; Tax et al. 1995; Van Wezel et al. 1997; Yang and Stein 1990).

It has been assumed that the phase-dependent response modulation adapts the responses in a functional way to the circumstances at various times in the step cycle. Previous studies have suggested that the phase-dependent responses and the corresponding joint angle changes following selective cutaneous stimulation might be functionally relevant in stumbling reactions (Van Wezel et al. 1997; Zehr et al. 1997). However, the EMG and the accompanying kinesiologic responses occurring after more realistic perturbations (e.g., stumbling over an object, such as a doorstep or a paving stone) have not been studied very extensively. Hence it is of importance to describe the compensatory reactions during stumbling and to study the functional significance of the observed responses.

To evoke natural stumbling reactions in an experimental setting, mechanical perturbations were induced by an obstacle suddenly rising above the ground and perturbing the forward swinging foot of subjects walking on a walkway (Eng et al. 1994). When a perturbation occurred in early swing, an "elevating strategy" was performed, during which the flexion angles of the hip, knee, and ankle of the swinging leg increased after the perturbation. In contrast, during late swing, mostly a "lowering strategy" was performed, in which the foot of the swinging leg was rapidly lowered to the ground causing a shortened step length. The reflex responses in the leg muscles during these recovery strategies had latencies varying from 60 to $140 \mathrm{~ms}$.

It cannot be determined whether the responses described above were mainly related to the phase of perturbation in the step cycle or to the strategy performed. Hence a method was developed in which the perturbations can be induced in all parts of the swing phase, including mid swing, in which both strategies could occur (Schillings et al. 1999a), allowing for a comparison of the two strategies in the same phase. Perturba- 
tions are caused by an obstacle put on a treadmill, which unexpectedly obstructs the forward swinging foot (Schillings et al. 1996). Because of its weight $(2.2 \mathrm{~kg})$, the obstacle has a considerable impact on the ongoing movement of the forward swinging leg. A previous study showed that after perturbations with this obstacle, short-latency stretch reflexes form a consistent part of the stumbling reactions (Schillings et al. 1999b). The aim of the present study is to describe the responses with longer latencies and the coordination of leg muscle activity compensating for this natural unexpected perturbation. The questions whether these responses are dependent on the phase of perturbation in the step cycle and/or whether the responses are functionally related to the stumble strategy performed (elevating or lowering) will be discussed.

\section{METHODS}

Eight healthy subjects ( 5 male, 3 female) between 20 and $47 \mathrm{yr}$ of age (mean age 27) participated in the experiment. They had no known history of neurological or motor disorder. The experiments were carried out in conformity with the declaration of Helsinki for experiments on humans. All subjects gave informed consent, and the study was approved by the local ethical committee.

\section{Experimental setup}

A detailed account of the experimental setup can be found in Schillings et al. (1996). While subjects walked on a treadmill (speed, $4 \mathrm{~km} / \mathrm{h}$ ), an obstacle (length, width, and height, $40.0,30.0$, and $4.5 \mathrm{~cm}$, respectively; weight, $2.2 \mathrm{~kg}$ ) was held by an electromagnet above the treadmill in front of the subject (Fig. 1A). To induce perturbations, the obstacle was dropped on the belt, thereby unexpectedly obstructing the forward sway of the left (ipsilateral) leg. Release of the obstacle occurred at a predetermined delay after ipsilateral or contralateral heel strike. In the thin flexible shoes, the toes were covered with a piece of cotton to protect them. A pressure-sensitive strip attached to the front of the obstacle measured the time at which the foot hit the obstacle. The subjects wore a pair of glasses, which blocked downward sight (and thus blocked the view of the obstacle). Earplugs eliminated most of the sound perception of the obstacle landing on the treadmill. In addition, the sound was masked by music through headphones. Further, to avoid that the subjects could feel the vibration of the obstacle landing on the treadmill, a heavy metal object was put on the treadmill at irregular intervals (imitating the landing of the obstacle). As a result of these measures, subjects were not able to perceive the obstacle before the collision with the foot. Subjects were instructed to keep the same position on the treadmill before the perturbation, but after the collision they were free to react without restrictions. The subjects wore a safety harness, fixed to a safety brake on the ceiling that would hold the subject and stop the treadmill in case a subject should start to fall. In practice, this never occurred because none of the subjects really started to fall. The harness was loosely suspended and did not provide extra stability during the experiment.

\section{Data sampling}

Bipolar surface electromyogram (EMG) activity of the biceps femoris (BF), rectus femoris (RF), tibialis anterior (TA), and soleus (SO) of both legs was measured. Laterally placed goniometers were used to measure the joint angles of the knee and ankle of the ipsilateral leg. Thin insole foot switches measured foot contact with the treadmill. Data were sampled in a time interval starting $100 \mathrm{~ms}$ before triggering the electromagnet and lasting for 2,100 ms. For the control trials the same intervals were sampled, but no obstacle was dropped after the trigger. The EMG was (pre-)amplified, high-pass filtered $(>3 \mathrm{~Hz})$, full-wave rectified, low-pass filtered $(<300 \mathrm{~Hz}), \mathrm{AD}$-converted $(500$
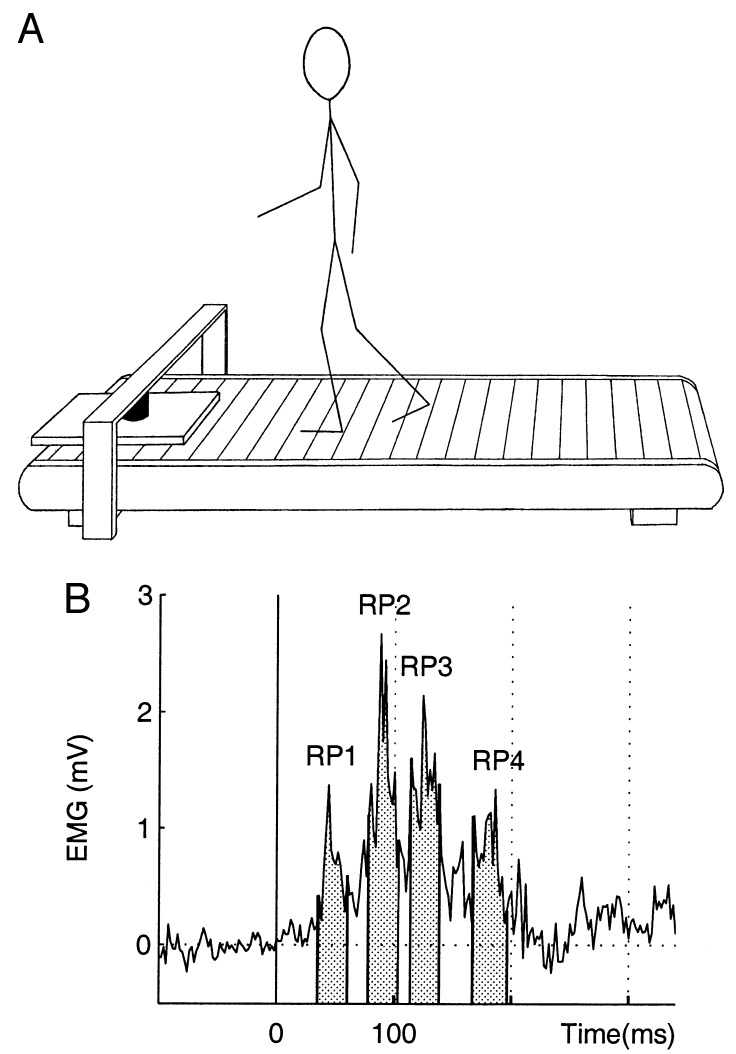

FIG. 1. Experimental methods. A: the electromagnet (black object) holds the obstacle above the treadmill in front of the subject's left (ipsilateral) foot (reprinted from Schillings et al. 1996, with permission from Elsevier Science). $B$ : example of an averaged subtracted electromyographic (EMG) response of the tibialis anterior during stumbling (averaged stumbling response minus averaged control EMG) showing the 4 response peaks (RP1-RP4). These 4 response peaks were not always clearly observable in every muscle during all phases. Window settings, which were used to determine the mean response amplitudes in this example, are indicated with gray. Zero time is the time the foot collided with the obstacle.

$\mathrm{Hz}$ ), and stored on hard disk along with the signals of the goniometers, foot switches, and pressure-sensitive strip. In practice, this sampling rate appeared to be sufficiently high. Increasing the sampling rate to $1,000 \mathrm{~Hz}$ did not lead to appreciable improvement of the signals for the purpose of this study. In addition, the subjects were recorded on video $(25 \mathrm{~Hz})$.

\section{Experimental protocol}

Each experiment consisted of three parts. Part one ( $5 \mathrm{~min})$ consisted of the registration of unperturbed walking. This control condition enabled to check whether the presentation of the obstacles (in following parts) affected baseline-walking characteristics (because of possible effects due to anticipation or fear of stumbling).

In part two $(20 \mathrm{~min})$, the effect of the timing of the perturbation on the behavioral response "strategies" (elevating or lowering) was studied for a wide variety of delays after onset of swing. For this purpose the computer triggered the electromagnet to drop the obstacle on the treadmill after fixed delays $(0,40,80, \ldots, 600 \mathrm{~ms})$ after heel strike. Each delay condition was randomly applied only once. A perturbation-free period of at least $10 \mathrm{~s}$ was taken between two succeeding trials to be sure that the subject was walking normally again at the time of the next perturbation. The normal walking pattern was usually regained within approximately two step cycles. The behavioral responses were classified in two categories (Fig. 2) on the basis of video analysis. A response was labeled as "elevating strategy" (Eng et al. 


\section{A Elevating strategy}

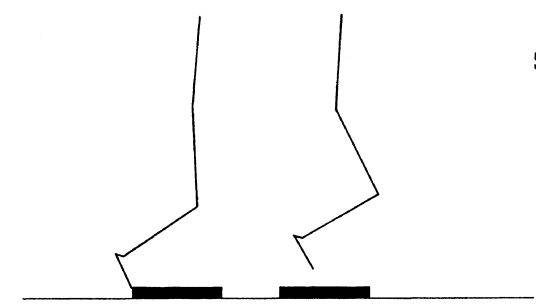

\section{B Lowering strategy}

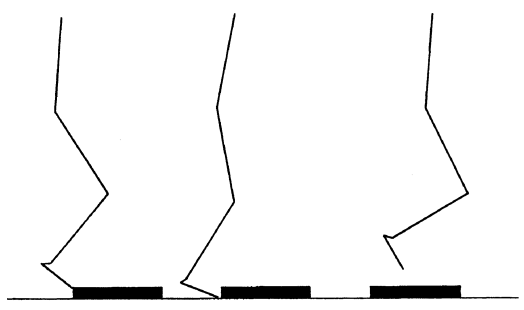

\section{Strategy as a function of perturbation onset}

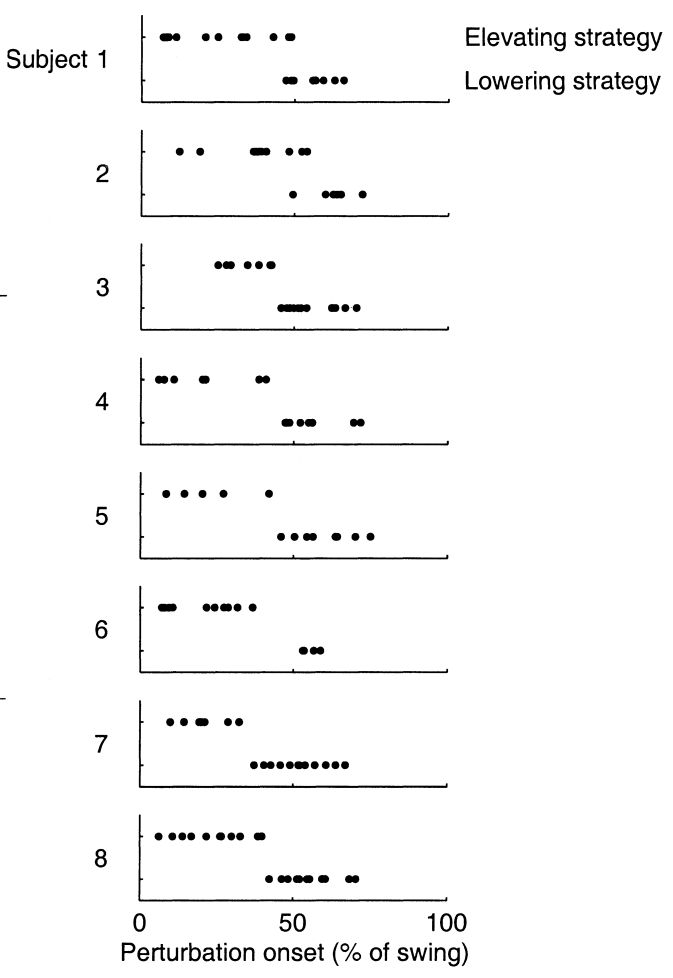

FIG. 2. Behavioral response strategies as a function of perturbation onset. $A$ : schematic picture of the characteristics of the foot trajectory during the elevating strategy. After the collision with the obstacle, the foot was directly lifted over the obstacle during the perturbed swing. $B$ : characteristics of the foot trajectory during the lowering strategy. After the perturbation, the ipsilateral foot was quickly placed on the treadmill without clearing the obstacle. The foot was lifted over the obstacle in the swing phase that succeeded the perturbed swing. $C$ : strategies performed as a function of perturbation onset in all subjects (\% of swing indicates time of obstacle contact with respect to control swing duration). These data are based on the 2nd part of the experiment (see METHODS).
1994) when the ipsilateral foot was lifted over the obstacle during the perturbed swing. The stumble response was classified as "lowering strategy" when the foot was first placed on the treadmill and then lifted over the obstacle.

In part three $(30 \mathrm{~min})$ stumbling reactions were repeatedly and randomly introduced during early swing $(5-25 \%$, time of obstacle contact with respect to control swing duration), mid swing (30-50\%), and late swing (55-75\%) to construct averages. On average eight trials (minimal 5 trials) were obtained for each phase of perturbation. The responses during these perturbed cycles were compared with unperturbed control trials obtained in between the perturbation trials (perturbation-free period between trials $>10 \mathrm{~s}$ ).

\section{Data analysis}

The stumble responses of each subject occurring in the same phase of the step cycle were averaged. In addition, the corresponding control trials were averaged. Then the averaged control activity was subtracted from the averaged stumbling trials. To quantify the amplitudes of the responses, the mean EMG activity was calculated in the period between the beginning and end of the response. For this purpose, windows were set around the individual response peaks occurring within the first $200 \mathrm{~ms}$ (see Fig. 1B). To enable a proper intersubject comparison of the response amplitudes, the resulting data of each muscle were normalized with respect to the maximal EMG activity during the control step cycles. The normalized responses of all subjects were averaged. This type of analysis was performed on four response peaks, namely RP1 (latency $\sim 40 \mathrm{~ms}$ ), RP2 (latency $\sim 75$ $\mathrm{ms}$ ), RP3 (latency $\sim 110 \mathrm{~ms}$ ), and RP4 (latency $\sim 160 \mathrm{~ms}$, see Fig. $1 B)$. The RP1 responses have already been described in a previous publication (Schillings et al. 1999b) and are only included in the present paper as a basis for comparison with the later responses. The Wilcoxon matched-pairs signed-rank test was used to test whether the response amplitudes during stumbling were significantly different from the control EMG activity. The Friedman two-way ANOVA was used to test whether the subtracted response amplitudes were different for the three phases of perturbation ( 8 triples of comparison: 8 subjects, 3 phases; $P<0.05$ ). The Wilcoxon rank sum test was used to compare the response amplitudes of the two strategies in mid swing $(P<0.05)$. The choice for nonparametric tests was based on the low number of averages that were compared (8 subjects).

\section{RE S U L T S}

\section{Normal walking}

To check whether the normal walking pattern was affected by the knowledge that a stumble over an obstacle could occur, the normal walking pattern measured in between the stumble trials (control trials of part 3) was compared with the normal walking pattern measured during unperturbed walking (part 1, see METHODS). None of the subjects clearly changed his/her normal walking pattern during the stumble experiment.

\section{Strategies in general}

The choice for the behavioral strategy depended on the timing of the perturbation in the step cycle. This is shown for all subjects in Fig. $2 C$ (data of 2 nd part of the experiment). When perturbations were caused in early swing $(5-25 \%$, time of obstacle contact with respect to control swing duration), all subjects showed the elevating strategy (see Fig. 2A). After perturbations in late swing $(55-75 \%)$, all subjects showed the lowering strategy (see Fig. $2 B$ ). In mid swing (30-50\%) both strategies could occur. It can be seen that some subjects showed a distinct transition in choice from elevating to lowering strategy (subjects 3-5, 7, and 8, Fig. 2C), whereas others showed a zone of overlap of the two strategies (for example, $49-54 \%$ in subject 2; Fig. 2C). For each subject the transition 
Elevating strategy (early swing)

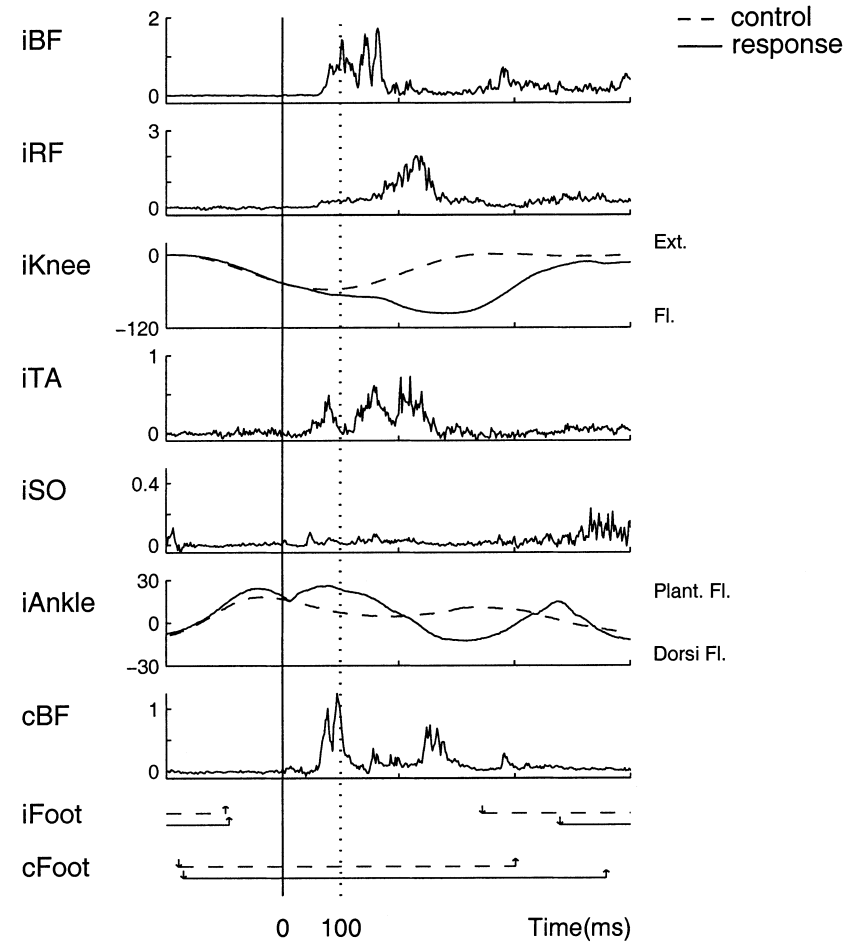

FIG. 3. Typical average subtracted EMG responses and joint angle changes during the early swing elevating strategy ( $n=8$ trials, subject 4$)$. EMG responses $(\mathrm{mV})$ are shown for the ipsilateral biceps femoris (iBF), rectus femoris (iRF), tibialis anterior (iTA), and soleus (iSO) as well as for the contralateral biceps femoris (cBF). Joint angle changes (degrees; not subtracted) are shown for the ipsilateral knee (iKnee) and ankle (iAnkle). Angle at standing position is zero. Two bottom traces show stance phases of the ipsilateral (iFoot) and contralateral foot (cFoot). Goniometer and foot signals: - , stumble responses; --- , control data $(n=15)$. Zero time is the time the foot collided with the obstacle. Ext., extension; Fl., flexion; Plant. Fl., plantar flexion; Dorsi Fl., dorsiflexion.

point from the elevating to the lowering strategy was defined as the time for one-half the interval between the last elevating and the first lowering strategy occurrences. This transition point varied for all subjects from $35 \%$ into the swing phase (subject 7, Fig. $2 C$ ) to $52 \%$ (subject 2, Fig. $2 C$ ) and was on average $44 \pm 5 \%$ (mean $\pm \mathrm{SD}, n=8$ subjects).

\section{Passive joint movements during both the elevating and the lowering strategy}

During both the elevating and the lowering strategy, the collision of the foot with the obstacle induced small passive movements in the ipsilateral knee and ankle joint. These movements are considered to be passive because they start before the occurrence of the first EMG responses in the muscles that could influence these joints. As seen in both the elevating strategy of Fig. 3 and the lowering strategy of Fig. 4 the ankle is first plantar flexed due to the collision (amplitude for all subjects between 1 and $10^{\circ}$ ) with a latency of $\sim 15 \mathrm{~ms}$, whereas the first responses in ipsilateral soleus and tibialis anterior (iSO and iTA) occurred with a latency of $\sim 40 \mathrm{~ms}$. Second, the knee was flexed due to the collision with a latency of $46 \mathrm{~ms}$ and an amplitude of $13^{\circ}$ during the elevating strategy (see Fig. 3) and $34 \mathrm{~ms}$ and $9^{\circ}$ during the lowering strategy (see Fig. 4). In the muscles ipsilateral biceps femoris and rectus femoris (iBF and $i R F)$, which could influence the knee joint angle, no responses were observed before this early flexion.

\section{Responses during the early swing elevating strategy}

After the collision with the obstacle, active ipsilateral knee and ankle flexion assisted the elevation of the swing foot to step over the obstacle. The active knee flexion started $\sim 160 \mathrm{~ms}$ after perturbation onset (see sudden increase of the knee flexion in Fig. 3; mean latency of active knee flexion for all subjects was $169 \pm 47 \mathrm{~ms})$. The maximum knee flexion reached during the elevating swing was considerably larger than during normal swing (96 vs. $58^{\circ}$ for the subject of Fig. 3 ). The upper leg muscles typically showed first a large iBF burst (latency, $64 \mathrm{~ms}$; Fig. 3) assisting knee flexion, followed by a large iRF burst (latency, 154 ms; Fig. 3) extending the knee before touch down.

The ankle dorsiflexion started $90 \mathrm{~ms}$ after perturbation, and the maximum dorsiflexion reached during the movement over the obstacle was $\sim 17^{\circ}$ larger than the maximum dorsiflexion during the unperturbed swing (Fig. 3). Facilitatory iTA responses (latency, $\sim 75 \mathrm{~ms}$ ) assisted this dorsiflexion. In two of eight subjects, suppressive iTA responses (latency, $\sim 80 \mathrm{~ms}$; suppressive response: the response EMG activity is lower than the control EMG activity) preceded the main facilitatory responses in iTA (latency, 112 and $214 \mathrm{~ms}$ ). This suppression possibly had the function to allow for ankle plantar flexion to avoid that the foot got hooked behind the obstacle. The change in foot trajectory caused a lengthening of the ipsilateral swing

Lowering strategy (late swing)

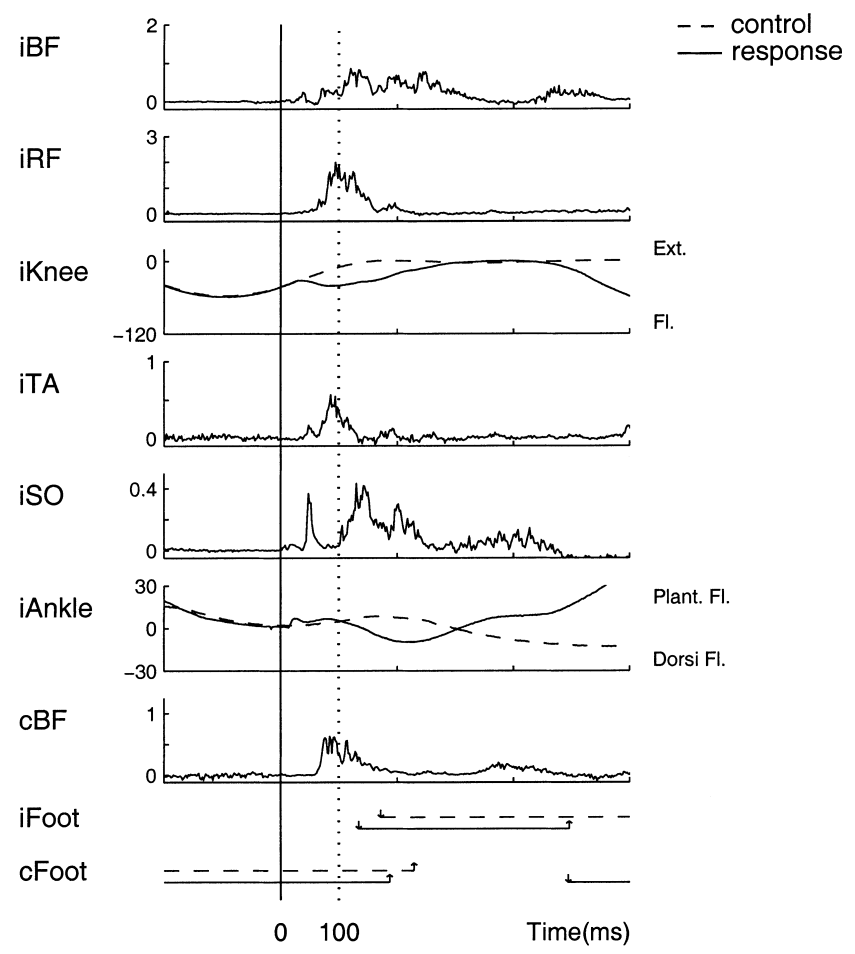

FIG. 4. Typical average subtracted EMG responses $(\mathrm{mV})$ and joint angle changes (degrees) during the late swing lowering strategy $(n=10$ trials, subject 4). Two bottom traces: stance phases of the ipsilateral (iFoot) and contralateral foot (cFoot). The same format is used as in Fig. 3. 
TABLE 1. Changes in ipsilateral swing and contralateral stance phase durations

\begin{tabular}{|c|c|c|c|c|c|c|c|c|}
\hline & \multicolumn{2}{|c|}{ Early Swing Elevating } & \multicolumn{2}{|c|}{ Mid Swing Elevating } & \multicolumn{2}{|c|}{ Mid Swing Lowering } & \multicolumn{2}{|c|}{ Late Swing Lowering } \\
\hline & iSwing & cStance & iSwing & cStance & iSwing & cStance & iSwing & cStance \\
\hline Subject 1 & +71 & $+54(9)$ & +146 & $+81(8)$ & & & $-38^{*}$ & $-64(9)$ \\
\hline Subject 2 & +146 & $+40(6)$ & +207 & +40 (10) & & & -17 & $-118(10)$ \\
\hline Subject 3 & +117 & $+80(9)$ & +129 & $+72(9)$ & & & -48 & $-65(10)$ \\
\hline Subject 4 & +128 & $+149 \dagger(8)$ & +240 & +92(10) & & & -38 & $-42(10)$ \\
\hline Subject 5 & +120 & $+92(5)$ & +137 & $+65(9)$ & & & -76 & $-22(10)$ \\
\hline Subject 6 & +154 & $+38(8)$ & & & +1 & $-82(7)$ & +5 & $-57(7)$ \\
\hline Subject 7 & +159 & $+115(6)$ & & & +1 & $-97(7)$ & -32 & $-64(7)$ \\
\hline Mean & $+128 \pm 30$ & $+81 \pm 41$ & $+172 \pm 49$ & $+70 \pm 20$ & $+1 \pm 0$ & $-90 \pm 11$ & $-35 \pm 25$ & $-62 \pm 29$ \\
\hline
\end{tabular}

Values in Mean are the pooled averages of all subjects \pm SD; number in parentheses is number of times the stumble strategy was observed in each subject. Mean differences (in ms) between stumble and control trials (stumble minus control) of the ipsilateral swing-phase durations and the contralateral stance-phase durations. The data of early, mid, and late swing perturbations are shown for all subjects separately and averaged. For the stumble trials the ipsilateral swing (iSwing) is the swing in which the ipsilateral foot hits the obstacle, and the contralateral stance (cStance) is the corresponding stance phase of the contralateral leg. After mid swing perturbations, subjects 1-5 repeatedly performed an elevating strategy; whereas subjects 6 and 7 performed a lowering strategy (data of 3 rd part of the experiment). The number of trials was always between 5 and 10. * Based on 3 trials; $\dagger$ based on 1 trial because of the absence of some foot switch data, which was also the reason for excluding subject 8 ( $n=9$ trials for each phase).

and concomitant of the contralateral stance with on average $128 \pm 30 \mathrm{~ms}$ and $81 \pm 41 \mathrm{~ms}$, respectively (see Table 1 ).

Contralaterally, a large burst of activity in the cBF appeared with a latency of $66 \mathrm{~ms}$ (subject of Fig. 3) after the perturbation. Because, during stance the biarticular BF serves as a hip extensor (Winter 1987), the cBF response could contribute to stabilizing the upper body by the standing leg after perturbations of the swinging leg, as was also suggested by other authors (Dietz et al. 1986b; Eng et al. 1994). In the other contralateral muscles (cRF, cTA, and cSO), the responses were too variable or small to give a detailed description.

\section{Responses during the late swing lowering strategy}

During the lowering strategy, the foot was quickly placed on the treadmill by shortening the forward sway and slightly extending the knee. The knee extension needed to place the foot was small in comparison with the knee extension before touch down during normal walking (see Fig. 4). This was first, because the foot was already close to the treadmill at the time of the perturbation $(\sim 2-4 \mathrm{~cm}$ above the treadmill) and second because the position of the foot during the landing (forefoot or flat foot landing) was different from normal (heel landing). The knee extension started $94 \mathrm{~ms}$ after perturbation and was presumably related to the large iRF burst (latency, $62 \mathrm{~ms}$; see Fig. 4). For all subjects, the iRF burst occurred on average $53 \pm 36$ $\mathrm{ms}$ before the average foot placing in late swing. In three of eight subjects, responses in iBF occurred approximately simultaneously with the responses in iRF (see Fig. 4). In the other five subjects the onset of the main responses in the $\mathrm{iBF}$ occurred $\sim 25 \mathrm{~ms}$ later than the onset of the main iRF burst. The iBF activity could slow down the forward swing in preparation of the early foot placement (hip joint angles were not measured).

In the lower leg muscles, first short-latency responses occurred with a latency of $40 \mathrm{~ms}$ in both iTA and iSO, possibly transiently enhancing ankle-joint stiffness (see Schillings et al. 1999b). Subsequently, a large activity burst was observed in iTA (latency, $66 \mathrm{~ms}$ after perturbation; Fig. 4), which could participate in the ankle dorsiflexion (latency, $90 \mathrm{~ms}$; Fig. 4). A large iSO burst appeared with a latency of $111 \mathrm{~ms}$ and was well timed to take up body support during the preliminary stance phase. This sequence of iTA and iSO responses was observed in seven of eight subjects and could support an initial movement away from the obstacle (iTA activity and ankle dorsiflexion) followed by foot placement (iSO activity). The mean latency of the premature placing off all subjects was $125 \pm 35 \mathrm{~ms}$ after the collision. The ipsilateral swing phase and the contralateral stance phase were shortened with, on average, $35 \pm 25 \mathrm{~ms}$ and $62 \pm 29 \mathrm{~ms}$ (Table 1 ), respectively. Contralaterally, the main consistent responses occurred in the $\mathrm{cBF}$, which showed a large response with a latency of $62 \mathrm{~ms}$ (subject of Fig. 4).

\section{Responses during mid swing}

After mid swing perturbations (30-50\% of swing), both strategies could occur. For the mid swing elevating strategy, the major characteristics were the same as for the early swing elevating strategy (lengthening of swing phase duration, increased knee flexion and ankle dorsiflexion, first iBF activation then iRF activation, large iTA activity). However, some small differences could be observed. For example, the duration of the perturbed swing was lengthened in both phases, but the swing phase duration increased on average $55 \pm 42 \mathrm{~ms}$ more in mid swing than in early swing (average of subjects $1-5$, see Table 1). Comparing the mid swing lowering with the late swing lowering strategy, it was found that the foot was placed later after mid swing (mean latency, $246 \mathrm{~ms}$; subjects 5-7) than after late swing perturbations (mean latency, $125 \mathrm{~ms}$; subjects 1-7).

The differences between responses in early and late swing could be related to the strategy performed. However, some of these differences may be related more to variations in the timing of the perturbation within the step cycle ("phase dependency"; see INTRODUCTION) than to changes in strategy. This complication does not occur for some of the data related to mid swing perturbations. Three subjects performed an elevating strategy in one trial, whereas a lowering strategy was used in another trial, despite the same timing of the perturbation. Hence in these cases it was possible to study which parts of the responses were strictly coupled to either lowering or elevating strategy. In Fig. 5 the signals of an elevating (thin lines) and a lowering strategy (heavy lines) occurring in the same phase are compared. 


\section{Elevating versus lowering strategy (mid swing)}

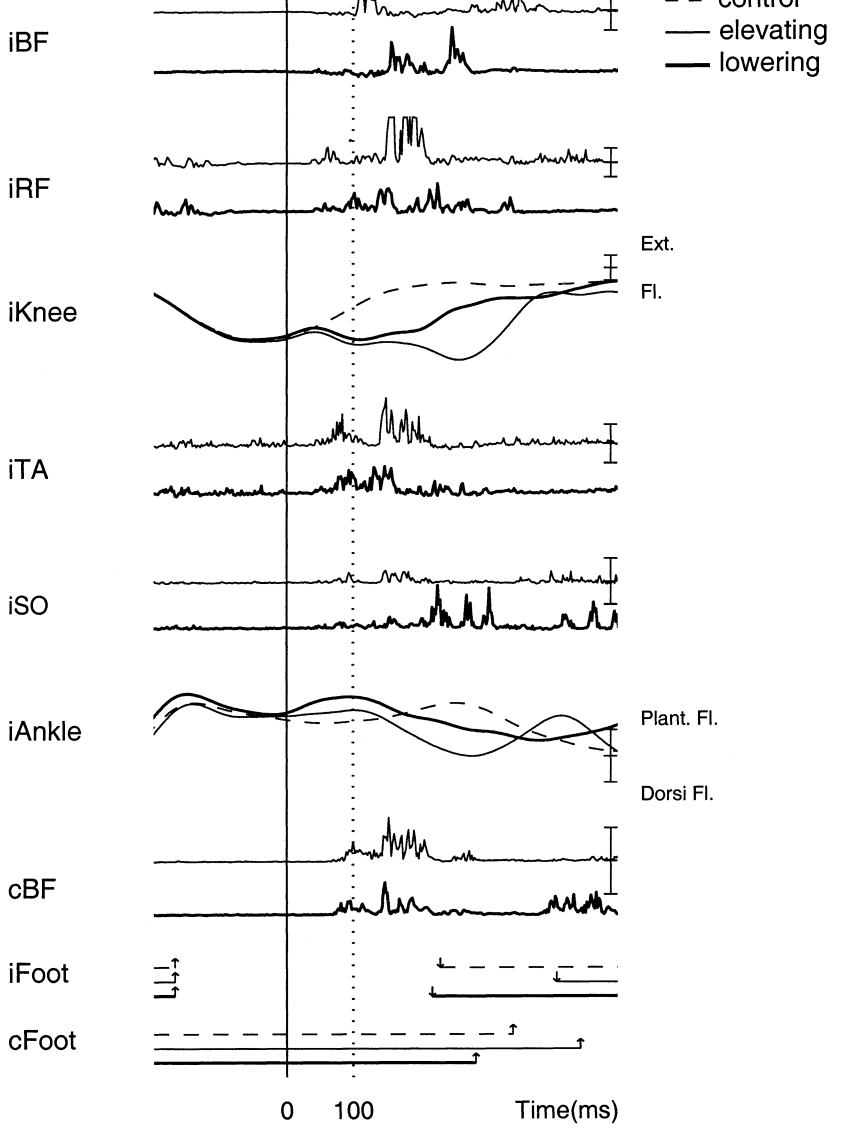

FIG. 5. Subtracted EMG responses $(\mathrm{mV})$ and averaged joint angles (degrees) of the elevating (thin lines) vs. the lowering strategy (heavy lines) after perturbations in mid swing for subject 5 (perturbation onset $41 \%$ of swing; data from 3rd part of the experiment). For each muscle 2 separate traces show the subtracted EMG responses for the 2 strategies. EMG calibration, $1 \mathrm{mV}$ (the calibration is the same for the 2 strategies). The joint angle changes (not subtracted) of the elevating strategy $(n=1)$, the lowering strategy $(n=1)$, and the averaged control (dashed line; $n=15$ trials) are superimposed. Angle at standing position is zero. Calibration goniometer traces, $10^{\circ}$. Two bottom traces show stance phases of the ipsilateral (iFoot) and contralateral foot (cFoot). Zero time is the time the foot collided with the obstacle. Ext., extension; Fl., flexion; Plant. Fl., plantar flexion; Dorsi Fl., dorsiflexion.

The knee goniometer signals of both the elevating and the lowering strategy started deviating from the control $(---)$ in the direction of knee flexion with a latency of $60 \mathrm{~ms}$. This knee flexion increased during both strategies till $\sim 150 \mathrm{~ms}$. After this common movement, the knee flexed further to lift the foot over the obstacle during the elevating strategy, whereas the knee started to extend to place the foot on the ground during the lowering strategy.

It was observed that the responses in the first $100 \mathrm{~ms}$ after the perturbation were similar for both strategies (Fig. 5). In this interval, the most obvious responses were observed in iTA with a latency of $\sim 70 \mathrm{~ms}$ in both the elevating and the lowering strategy. In the period after $100 \mathrm{~ms}$, the first difference between the two strategies occurred in iBF, namely after $104 \mathrm{~ms}$ (subject of Fig. 5). In this subject, the iBF (knee flexor) showed a burst, which was followed by a burst in the iRF (latency, 148 ms; Fig. 5) during the elevating strategy. In contrast, during the lowering strategy the iRF was activated (latency, $136 \mathrm{~ms}$; Fig. 5) before the iBF. Similar results were observed in two other subjects. In each case there was a common initial movement in the knee (mean onset of difference in knee trajectory was 136 and $186 \mathrm{~ms}$, respectively). Correspondingly, these two subjects showed a common pattern of EMG responses during the first $100 \mathrm{~ms}$ for the two strategies.

\section{Delayed lowering strategy}

In the previous section it was shown that the EMG responses during the first $100 \mathrm{~ms}$ did not determine the ensuing behavioral response (strategy). Later EMG bursts (between 100 and $150 \mathrm{~ms}$ ) were characteristic for the strategies, but the question remains how predetermined these later responses were. If these responses were completely defined from the moment of collision onward, a fixed response pattern could be expected and no changes should occur during the course of this reaction. The example shown in Fig. 6, however, illustrates that this is not the case. In this exceptional trial, the subject started with an elevating strategy after the early swing perturbation, but the obstacle stuck to the toes and the subject was unable to clear the obstacle. Instead, he extended the knee (latency, $236 \mathrm{~ms}$ ) and placed the foot on the treadmill (latency, $416 \mathrm{~ms}$ ) without

Elevating versus delayed lowering strategy (early swing)

$\mathrm{iBF}$

iRF

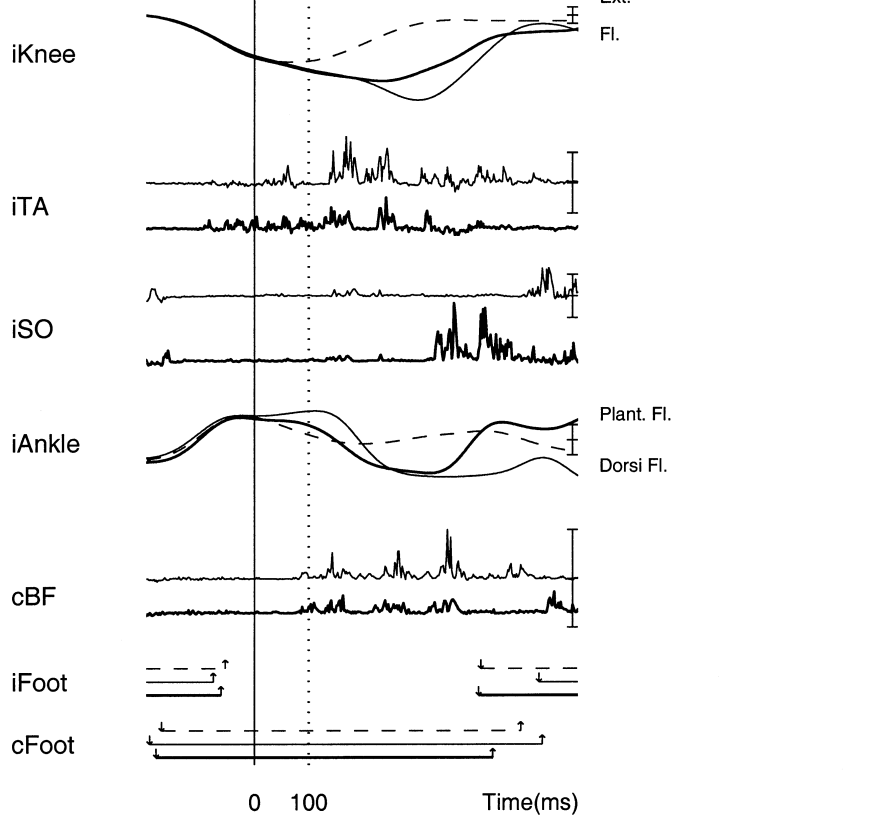

FIG. 6. Subtracted EMG responses $(\mathrm{mV})$ and averaged joint angles (degrees) of the elevating (thin lines, $n=1$ ) vs. the delayed lowering strategy (heavy lines, $n=1$ ) after perturbations in early swing for subject 6 (perturbation onset 16 and $14 \%$ of swing, respectively; data from 3rd part of the experiment). The same format is used as in Fig. 5. 
TABLE 2. Mean onset and end of windows around the four response peaks

\begin{tabular}{lcccc}
\hline \hline & RP1 & RP2 & RP3 & RP4 \\
\hline iSO & $42-67( \pm 3)$ & $76-100( \pm 4)$ & $112-147( \pm 6)$ & $162-197( \pm 12)$ \\
iTA & $41-64( \pm 3)$ & $78-105( \pm 7)$ & $103-143( \pm 3)$ & $164-197( \pm 3)$ \\
iBF & $34-53( \pm 4)$ & $71-98( \pm 5)$ & $104-140( \pm 5)$ & $159-200( \pm 8)$ \\
iRF & $39-63( \pm 4)$ & $72-94( \pm 6)$ & $118-151( \pm 10)$ & $160-198( \pm 7)$ \\
cBF & & $74-104( \pm 5)$ & $164-201( \pm 14)$ \\
\hline
\end{tabular}

Number in parentheses is \pm SD of the window onset. Mean onset and end of windows, which were set around the 4 response peaks (RP1-RP4) in the muscles iSO, iTA, iBF, iRF, and cBF. These values are means of all subjects $(n=8)$ in ms after perturbation. i, ipsilateral; SO, soleus; TA, tibialis; BF, biceps femoris; $\mathrm{RF}$, rectus femoris; c, contralateral.

clearing the obstacle ("delayed lowering"). In the succeeding swing phase the foot was lifted over the obstacle. In this situation, in which the reaction was in fact a mixture of the two strategies, the EMG responses of the two strategies (normal elevating and delayed lowering) showed similar responses till $\sim 120 \mathrm{~ms}$ after perturbation. From then on the iRF showed a large response during the delayed lowering strategy that was absent during the elevating strategy. This iRF burst occurred $116 \mathrm{~ms}$ before the onset of the knee extension, which resulted in foot placement.

\section{Modulation of the response amplitudes}

In the EMG traces the main responses of the stumbling reactions occurred with four peaks (RP1-RP4) within the first $200 \mathrm{~ms}$. To study the amplitudes of the EMG peaks, time windows were set in these four periods, and the mean EMG activity was calculated within these time windows (see Table 2).

In Fig. 7, the amplitudes of the mean normalized control and response activity of all subjects are shown during early swing elevating ( $n=8$ subjects), mid swing elevating $(n=5)$, mid swing lowering $(n=3)$, and late swing lowering reactions $(n=8)$. Below the bars is indicated whether the pooled average response amplitudes of all subjects were significantly different from the average control activity $(*)$ or not $(\bigcirc)$.

Representing the data of all subjects together (Fig. 7) enabled us to study phase and strategy dependency for the data of all subjects. First, to study whether different response peaks are phase dependent, the responses occurring in the three phases [early swing (ES) vs. mid swing (MS) vs. late swing (LS), Fig. 7] were compared, irrespective of the strategy performed. Almost all responses were phase-dependent as determined with the Friedman two-way ANOVA $(P<0.05)$. The only responses that did not show a significant phase effect were the RP2 of iSO, RP3 and RP4 of iBF, and RP1 and RP2 of iRF.

In general for all muscles, the response amplitudes were not strictly related to the background EMG activity (see Fig. 7). For a clear example, compare the response amplitudes of the iTA RP3 that showed largest responses in mid swing, when the background activity was lowest, and the smallest responses in late swing, when the background activity was largest.

Second, to study whether the responses are strategy dependent, the responses occurring during the two strategies [elevating (el) vs. lowering (lo), Fig. 7] in mid swing were compared. As expected on the basis of the observation of Fig. 5, no significant differences in the RP1 and RP2 amplitudes between the elevating and the lowering strategy in mid swing were observed (Wilcoxon rank sum test). The amplitudes of the late responses (RP4) in iSO were significantly related to the strategy. A general characteristic of the late iSO responses in mid swing was that they were small during the elevating strategy and large during the lowering strategy.

\section{I S C U S S I O N}

The present study demonstrates that stumbling over a rigid object on a treadmill induces a sequence of EMG and behavioral responses. The elevating and lowering stumbling strategies presently described show basic similarities with previously described stumbling reactions, which were induced by more flexible obstacles (Eng et al. 1994). However, some differences were observed. First, the short-latency stretch reflexes in the ipsilateral leg muscles were not present after perturbations with the flexible metal strip (Eng et al. 1994; Rietdyk and Patla 1998). The larger impact of the collision, influenced by the weight and flexibility of the obstacle, in the present study might be the cause of this difference. The occurrence of short-latency stretch reflexes in both flexors and extensors can be understood because these responses are caused by a sudden jar through the ipsilateral leg due to the collision of the foot with the obstacle (see Schillings et al. 1999b).

Second, in the late swing lowering strategy, the quick foot placement after the perturbation was accompanied by iTA and iRF facilitations (latency, $\sim 75 \mathrm{~ms}$ ). Eng et al. (1994) described suppressive responses during the same period in these muscles, and they speculated that this permitted gravity to assist the lowering of the foot. Again, the observed difference might be related to the characteristics of the perturbing obstacle. With the presently used obstacle, subjects tried to avoid that they would step on the obstacle. So they tended to move away from the obstacle (iTA activity) before the quick touchdown assisted by iRF. In the study of Eng et al. (1994), there was no chance to step on the obstacle, because the flexible metal strip disappeared directly after the collision (the strip turned to a flat position). Hence it was of less importance to actively control the quick foot placement.

Third, Eng et al. (1994) described a "reaching strategy" after late swing perturbations, whereas in the present study this strategy was not observed. During this strategy, the foot is directly lifted over the obstacle, primarily due to hip flexion rather than knee flexion. Apparently the reaching strategy was avoided in the present study because it requires considerably more hip flexion to cross a long obstacle than a short one. 


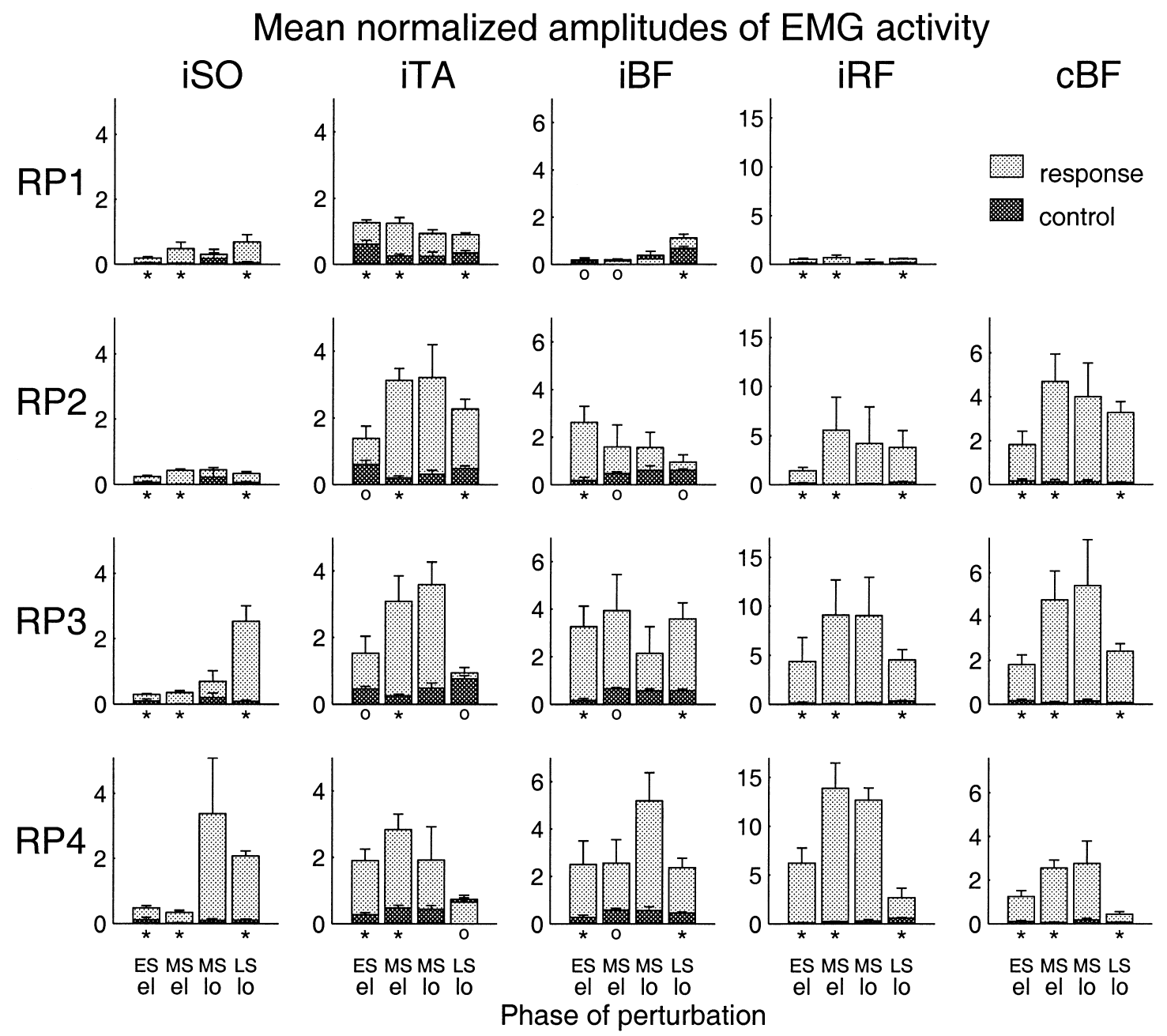

FIG. 7. Mean normalized EMG amplitudes (average of all subjects) of the 4 response peaks (RP1-RP4) in iSO, iTA, iBF, iRF, and $\mathrm{cBF}$. Response amplitudes are shown with respect to the phase of perturbation and with respect to the behavioral strategy: early swing elevating (ES el), mid swing elevating (MS el), mid swing lowering (MS lo), and late swing lowering (LS lo). Dark bars show the normalized background activity (normalization with respect to the maximum background locomotor activity). Light bars show the normalized EMG response amplitudes during the stumbling reactions. In this way, the part of the light bars above the dark bars indicate the amplitude of the subtracted responses. In case the response was suppressive, the dark bar was larger than the light bar (see, for example, iTA, LS lo, RP4). The SE of the control activity and the subtracted response activity is shown by error bars. Number of trials per subject: 5-10 (see Table 1 between brackets). Averaged responses were calculated by averaging the mean responses of all subjects: $n=8$ subjects for ES el and LS lo, $n=5$ for MS el, and $n=3$ for MS lo. *, pooled average response amplitudes of all subjects were significantly different from the averaged control activity (Wilcoxon matched-pairs signed-rank test, $P<0.05)$. $\bigcirc$, nonsignificant responses $(P>0.05)$. The responses of the mid swing lowering (MS lo) were not analyzed with the Wilcoxon matched-pairs signed-rank test $(P<0.05)$ because the number of subjects was too small $(n=3)$.

\section{Phase dependency of the EMG responses}

Differences in response amplitudes in the three phases (early, mid, and late swing) could either be due to changes in stimulation or to modulation of reflexes by the nervous system. Changes in the intensity of the collision of the foot with the obstacle could influence the amplitude of the EMG responses. The impact of the collision on the foot is mainly dependent on the horizontal toe velocity, which varies during the swing phase. The horizontal toe velocity is $\sim 1.5$ times lower in early swing than in either mid or late swing (Winter 1992). If the amplitudes of the responses were completely determined by the impact of the collision, one would always expect the smallest responses in early swing. This was not the case. For example, the RP2 response of iBF was largest in early swing and smallest in late swing (see Fig. 7). Furthermore, one would expect about equal amplitudes in mid and late swing. However, different response amplitudes were often observed in these two phases. Another possible cause for the amplitude modulation observed could be that the responses were related to the background activity (Matthews 1986). However in general, there was no strict relation between reflex amplitude and background activity. This leaves the possibility that premotoneuronal mechanisms might contribute to the phase-dependent modulation of the response amplitudes.

\section{Initial common reaction of the two strategies in mid swing}

The occurrence of the two strategies after perturbations in mid swing offered the unique possibility to study which parts of the responses were strictly coupled to either the lowering or the elevating strategy. Both strategies in mid swing started with 
the same EMG responses during the first $100 \mathrm{~ms}$ after the perturbation. Neither the RP1 nor the RP2 responses predicted the choice for one of the two strategies. Only the RP3 and RP4 responses $(>100 \mathrm{~ms})$ of some muscles (iBF and iSO) correlated well with the behavioral strategy performed (e.g., see the large iBF RP3 during the mid swing elevating strategy in Fig. 5 , and see the large iSO RP4 during the mid swing lowering strategy in Fig. 7). The initial common reaction of the two strategies in mid swing first consists of the short-latency stretch reflex (RP1), which might contribute to a temporary stiffening of the joint (Schillings et al. 1999b). Second, it involves the RP2 response in muscles such as iTA. The iTA RP2 activation could contribute to the observed ankle dorsiflexion to move the foot away from the obstacle. The same initial reaction of the two strategies possibly provides the CNS sufficient time to integrate information obtained by various sensory receptors (Brooke et al. 1997; Dietz 1992; Jankowska 1992) and supraspinal sources (see review Dietz 1992) to make an appropriate decision about the final behavioral strategy.

\section{Behavioral responses are not predetermined at the time of perturbation}

The occurrence of both behavioral strategies in mid swing indicates that the decision about the final behavioral strategy is not tightly linked to the time of impact. Further support for this idea comes from the example of a lowering strategy performed when a subject's foot got hooked behind the obstacle during an early swing perturbation. The foot was first lifted as in the elevating strategy (initial reaction plus onset of the elevating strategy), but because the obstacle stuck to the toes (continuing mechanoreceptor feedback information), the subject decided to place the foot on the ground again and finally completed a delayed lowering strategy. Apparently, on-line afferent information during the stumble response is integrated in the final reaction and can be used to adjust the strategy to the demands of the moment. In the example of the delayed lowering strategy, the earliest adaptive response to extend the leg for the foot placement on the treadmill was observed after $120 \mathrm{~ms}$ in the $\mathrm{iRF}$. This is too early to be a voluntary reaction, because the earliest EMG changes during voluntary reactions occur after $\sim 150 \mathrm{~ms}$. For example, in a study of Hase and Stein (1998), in which subjects were instructed to stop walking as soon as they got a cue by electrical stimulation of the superficial peroneal nerve, the earliest voluntary changes in EMG activity of leg muscles occurred 150-200 ms after stimulation.

The idea that a corrective response can be adjusted en route has found support in some earlier observations as well. While subjects were walking on a treadmill, Dietz et al. (1986a) applied a holding impulse by a cord attached to the swinging leg, which was followed by a second perturbation, i.e., a treadmill deceleration. On the basis of their results, they suggested that the first part of the compensatory reaction is released as an immutable pattern within the spinal cord. In contrast, the later part of the response (in the order of $120 \mathrm{~ms}$ after the 1st perturbation, see their Fig. 2) can be modified by external factors and adjusted to the actual nature of the task.

Furthermore, the present data are in line with the finding of bimodal responses in subjects who were tripped while they were taking a single step forward (Rietdyk and Patla 1998). In this situation, the perturbation always induced an initial change in ankle trajectory (elevation of the ankle), which could or could not be followed by a second elevation of the ankle. Thus the initial response could be followed by a later correction, which resulted in an enhancement of the initial movement. In the present study it was demonstrated that the later correction is not always an enhancement of the first movement as observed in the study of Rietdyk and Patla (1998), but can also be a reversal of the first movement.

\section{Origin of the responses}

On the basis of several studies on cats, it has been assumed that the responses observed during "stumbling corrective reactions" (Forssberg 1979) are mainly cutaneous in origin (Forssberg 1979; Forssberg et al. 1975, 1977; Prochazka et al. 1978; Wand et al. 1980). In addition, for humans it has been suggested that the medium-latency EMG responses and the accompanying joint angle changes after electrical cutaneous stimulation might be functionally relevant in the context of stumbling reactions (Van Wezel et al. 1997; Zehr et al. 1997; see, however, Duysens et al. 1992). There are indeed some similarities between the modulation of medium-latency cutaneous responses and the RP2 responses observed during stumbling. For example, the iTA facilitation (RP2) with ankle dorsiflexion observed during the early swing elevating strategy was also observed after sural nerve stimulation in early swing (Duysens et al. 1992; Van Wezel et al. 1997). However, cutaneous stimulation evoked suppression of the iTA activity in late swing (Duysens et al. 1990; Yang and Stein 1990; Zehr et al. 1997), whereas the present mechanical perturbation evoked facilitatory iTA (RP2) responses. Hence these differences indicate that the RP2 observed during stumbling in humans cannot be fully attributed to cutaneous responses (although we cannot rule out the possibility that cutaneous responses after mechanical perturbations are different from cutaneous responses after electrical stimulation).

Alternatively, proprioceptive afferents might contribute to the RP2 responses observed. Medium-latency stretch responses (M2 or MLR) in leg muscles with latencies similar to the RP2 responses $(\sim 75 \mathrm{~ms})$ have been described by many authors after joint rotations during various conditions (Fellows et al. 1993; Nielsen et al. 1998; Schieppati and Nardone 1997; Schieppati et al. 1995; Sinkjaer et al. 1988; Toft et al. 1989). Although some authors suggested that Ia afferents could mediate medium-latency stretch reflexes in leg muscles (Berardelli et al. 1982; Fellows et al. 1993), most evidence points in the direction of a contribution of muscle proprioceptive group II afferents in these responses (see Corna et al. 1995; Dietz 1992; Nardone et al. 1996; Nielsen et al. 1998; Schieppati and Nardone 1997; Schieppati et al. 1995). Even activations of Ib afferents cannot be excluded because of the strong impact of the obstacle (for review see Duysens et al. 2000). The contribution of vestibular afferents in the RP2 responses during stumbling is probably small because vestibular responses have much smaller amplitudes than somatosensory responses (Horstmann and Dietz 1988).

For the short-latency responses, there is little doubt that these are spinal stretch reflexes mediated by Ia afferents (see Schillings et al. 1999b). The responses with longer latencies (RP2-RP3) during stumbling could follow both spinal or supraspinal neural pathways. In principle, these responses might 
be polysynaptic EMG responses of spinal origin and could be related to activation of slower conducting afferents (see review Dietz 1992). However, the latency of these responses is also long enough to be compatible with long-loop reflexes through the cortex (Christensen et al. 1999; Nielsen et al. 1997; Petersen et al. 1998; Pijnappels et al. 1998). Although the same neural pathways could contribute to the RP4 responses, these responses are likely to be at least partly under voluntary control because they have latencies above the voluntary reaction time of $\sim 150 \mathrm{~ms}$ in leg muscles.

We thank P.H.J.A. Nieuwenhuijzen and H.W.A.A. Van de Crommert for help with the experiments and the analysis software. We also acknowledge G. Windau for developing the software and A. M. Van Dreumel and J.W.C. Kleijnen for technical assistance.

This study was supported by the Dutch Science Foundation (NWO).

Address for reprint requests: A. M. Schillings, Dept. of Medical Physics and Biophysics, University of Nijmegen, P.O. Box 9101, NL-6500 HB Nijmegen, The Netherlands.

Received 9 August 1999; accepted in final form 13 December 1999.

\section{REFERENCES}

Berardelli, A., Hallett, M., Kaufman, C., Fine, E., Berenberg, W., and Simon, S. R. Stretch reflexes of triceps surae in normal man. J. Neurol. Neurosurg. Psychiatry 45: 513-525, 1982.

Brooke, J. D., Cheng, J., Collins, D. F., Mcllroy, W. E., Misiaszek, J. E., and Staines, W. R. Sensori-sensory afferent conditioning with leg movement: gain control in spinal reflex and ascending paths. Prog. Neurobiol. 51: 393-421, 1997.

Christensen, L. O., Morita, H., Petersen, N., and Nielsen, J. Evidence suggesting that a transcortical reflex pathway contributes to cutaneous reflexes in the tibialis anterior muscle during walking in man. Exp. Brain Res. 124: 59-68, 1999.

Corna, S., Grasso, M., Nardone, A., And Schieppati, M. Selective depression of medium-latency leg and foot muscle responses to stretch by an $\alpha_{2}$-agonist in humans. J. Physiol. (Lond.) 484: 803-809, 1995.

DIETZ, V. Human neuronal control of automatic functional movements: interaction between central programs and afferent input. Physiol. Rev. 72: 33-69, 1992.

DietZ, V., Quintern, J., AND BERGER, W. Stumbling reactions in man: release of a ballistic movement pattern. Brain Res. 362: 355-357, 1986 .

Dietz, V., Quintern, J., Boos, G., AND Berger, W. Obstruction of the swing phase during gait: phase-dependent bilateral leg muscle coordination. Brain Res. 384: 166-169, 1986b.

Duysens, J., Clarac, F., AND Cruse, H. Load regulating mechanisms in gait and posture: comparative aspects. Physiol. Rev. 80: 83-133, 2000.

Duysens, J., TAX, A.A.M., Murrer, L., AND DiETZ, V. Backward and forward walking use different patterns of phase-dependent modulation of cutaneous reflexes in humans. J. Neurophysiol. 76: 301-310, 1996.

Duysens, J., Tax, A.A.M., Trippel, M., and Dietz, V. Phase-dependent reversal of reflexly induced movements during human gait. Exp. Brain Res. 90: 404-414, 1992.

Duysens, J., Trippel, M., Horstmann, G. A., and Dietz, V. Gating and reversal of reflexes in ankle muscles during human walking. Exp. Brain Res. 82: 351-358, 1990.

EnG, J. J., Winter, D. A., AND PATLA, A. E. Strategies for recovery from a trip in early and late swing during human walking. Exp. Brain Res. 102: 339-349, 1994.

Fellows, S. J., Dömges, F., Töpper, R., Thilmann, A. F., And Noth, J. Changes in the short- and long-latency stretch reflex components of the triceps surae muscle during ischaemia in man. J. Physiol. (Lond.) 472: 737-748, 1993.

ForsSBERG, H. Stumbling corrective reaction: a phase-dependent compensatory reaction during locomotion. J. Neurophysiol. 42: 936-953, 1979.

Forssberg, H., Grillner, S., AND Rossignol, S. Phase dependent reflex reversal during walking in chronic spinal cats. Brain Res. 85: 103-107, 1975.

ForssberG, H., Grillner, S., AND Rossignol, S. Phasic gain control of reflexes from the dorsum of the paw during spinal locomotion. Brain Res. 132: 121-139, 1977.
Hase, K. and Stein, R. B. Analysis of rapid stopping during human walking. J. Neurophysiol. 80: 255-261, 1998.

Horstmann, G. A. And Dietz, V. The contribution of vestibular input to the stabilization of human posture: a new experimental approach. Neurosci. Lett. 95: 179-184, 1988.

JANKOWSKA, E. Interneuronal relay in spinal pathways from proprioceptors. Prog. Neurobiol. 38: 335-378, 1992.

MAtTHEws, P.B.C. Observations on the automatic compensation of reflex gain on varying the pre-existing level of motor discharge in man. J. Physiol. (Lond.) 374: 73-90, 1986.

Nardone, A., Grasso, M., Giordano, A., AND Schieppati, M. Different effect of height on latency of leg and foot short- and medium-latency EMG responses to perturbation of stance in humans. Neurosci. Lett. 206: 89-92, 1996.

Nielsen, J., Petersen, N., ANd FedirchuK, B. Evidence suggesting a transcortical pathway from cutaneous foot afferents to tibialis anterior motoneurones in man. J. Physiol. (Lond.) 501: 473-484, 1997.

Nielsen, J., Sinkjaer, T., Baumgarten, J., Andersen, J. B., Toft, E., ChrisTENSEN, L.O.D., LAdoceur, M., AND MoRita, H. Modulation of the soleus stretch reflex and H-reflex during human walking after block of peripheral feedback. Soc. Neurosci. Abstr. 24: 2103, 1998.

Petersen, N., Christensen, L.O.D., Morita, H., Sinkjaer, T., And Nielsen, J. Evidence that a transcortical pathway contributes to stretch reflexes in the tibialis anterior muscle in man. J. Physiol. (Lond.) 512: 267-276, 1998.

Pijnappels, M., Van Wezel, B.M.H., Colombo, G., Dietz, V., and Duysens, J. Cortical facilitation of cutaneous reflexes in leg muscles during human gait. Brain Res. 787: 149-153, 1998.

Prochazka, A., SonTAG, K. H., AND WAND, P. Motor reactions to perturbations of gait: proprioceptive and somesthetic involvement. Neurosci. Lett. 7: 35-39, 1978.

Rietdyk, S. And PatLa, A. E. Context-dependent reflex control: some insights into the role of balance. Exp. Brain Res. 119: 251-259, 1998.

SChIEPPATI, M. AND NARDONE, A. Medium-latency stretch reflexes of foot and leg muscles analysed by cooling the lower limb in standing humans. J. Physiol. (Lond.) 503: 691-698, 1997.

Schieppati, M., Nardone, A., Siliotto, R., and Grasso, M. Early and late stretch responses of human foot muscles induced by perturbation of stance. Exp. Brain Res. 105: 411-422, 1995.

Schillings, A. M., Van Wezel, B.M.H., and Duysens, J. Mechanically induced stumbling during human treadmill walking. J. Neurosci. Methods 67: 11-17, 1996.

Schillings, A. M., Van Wezel, B.M.H., Mulder, Th., and Duysens, J. Stumbling and stepping over obstacles during human treadmill walking in young and older adults (Abstract). Gait Pos. 9: S30, 1999a.

Schillings, A. M., Van Wezel, B.M.H., Mulder, Th., and Duysens, J. Widespread short-latency stretch reflexes and their modulation during stumbling over obstacles. Brain Res. 816: 480-486, 1999b.

Sinkjaer, T., Toft, E., Andreassen, S., and Hornemann, B. C. Muscle stiffness in human ankle dorsiflexors: intrinsic and reflex components. J. Neurophysiol. 60: 1110-1121, 1988.

TAX, A.A.M., VAN Wezel, B.M.H., AND DieTZ, V. Bipedal reflex coordination to tactile stimulation of the sural nerve during human running. J. Neurophysiol. 73: 1947-1964, 1995.

Toft, E., SinkJaer, T., AND Andreassen, S. Mechanical and electromyographic responses to stretch of the human anterior tibial muscle at different levels of contraction. Exp. Brain Res. 74: 213-219, 1989.

Van WeZel, B.M.H., OtTenhoff, F.A.M., And Duysens, J. Dynamic control of location-specific information in tactile cutaneous reflexes from the foot during human walking. J. Neurosci. 17: 3804-3814, 1997.

Wand, P., Prochazka, A., AND Sontag, K. H. Neuromuscular responses to gait perturbations in freely moving cats. Exp. Brain Res. 38: 109-114, 1980.

WinTER, D. A. The Biomechanics and Motor Control of Human Gait. Waterloo, Ontario: Univ. of Waterloo Press, 1987.

WINTER, D. A. Foot trajectory in human gait: a precise and multifactorial motor control task. Phys. Ther. 72: 45-56, 1992.

YANG, J. F. AND Stein, R. B. Phase-dependent reflex reversal in human leg muscles during walking. J. Neurophysiol. 63: 1109-1117, 1990.

Zehr, E. P., Komiyama, T., AND Stein, R. B. Cutaneous reflexes during human gait: electromyographic and kinematic responses to electrical stimulation. J. Neurophysiol. 77: 3311-3325, 1997. 